\section{Early versus late traumatic cataract surgery and intraocular lens implantation}

SA Tabatabaei ${ }^{1}$, MB Rajabi ${ }^{1}$, SM Tabatabaei ${ }^{1}$, M Soleimani ${ }^{1}$, F Rahimi ${ }^{1}$ and $M$ Yaseri $^{2}$

\begin{abstract}
Purpose To determine the proper time for traumatic cataract surgery after open globe injuries.
\end{abstract}

Setting Farabi Eye Hospital, Tehran

University of Medical Sciences, Tehran, Iran.

Design Randomized clinical trial.

Patients and methods In a randomized

clinical trial, 30 eyes with traumatic cataract

after open globe injury with IOL

implantation underwent early (in the first

week after the trauma) and 30 eyes underwent late cataract surgery (from the first to second month after the trauma). We excluded patients who were under 12-yearold. All patients were visited at 1 week, 4 weeks, 12 weeks, and 6 months after surgery. In each visit, patients were examined regarding visual acuity, intraocular pressure (IOP), anterior chamber inflammation, IOL position, and posterior synechiae. In addition, posterior segment evaluation and fundoscopy were performed. Intraoperative complication including posterior capsular rupture, anterior vitrectomy, and zonulysis as well as the site of IOL implantation were documented and post-operative complications including raised IOP, anterior chamber inflammation, visual axis opacity, posterior synechiae, subluxation of IOL, and IOL pigment deposition were listed.

Results Best-corrected visual acuity 6 months after surgery was not different between the two groups. Also in early cataract surgery group, the rate of posterior capsular rupture was not significantly higher than the late surgery group $(P=0.069)$. On the other hand, zonulysis was significantly higher in the late procedure group $(P=0.039)$. Other complications including anterior vitrectomy, raised IOP, anterior chamber inflammation, visual axis opacity, posterior synechiae, subluxation of IOL, and IOL pigment deposition were not different in the two groups.

Conclusions Early and late traumatic cataract surgery and IOL implantation after open globe injuries, have no significant difference regarding the post-surgical BCVA and prominent intraoperative and post-operative complications.

Eye (2017) 31, 1199-1204; doi:10.1038/eye.2017.57; published online 14 April 2017

Ocular disease and injuries represent a sizeable burden to emergency departments, have been associated with long-term sequelae and reduced quality of life. ${ }^{1,2}$ The Baltimore Eye Survey has reported a $14.4 \%$ cumulative lifetime prevalence of eye injury in the general population. ${ }^{3}$ Another study has indicated a 13.2 per 100000

population annual rate of hospitalization due to ocular trauma. ${ }^{4}$

Ocular trauma is frequently associated with cataractous changes in the lens. 5,6 Traumatic cataract is encountered in $27-65 \%$ of the ocular trauma cases. ${ }^{7-9}$ It has been reported that ocular trauma occurs in 3.45 per 100000 population per year or 4.6 per 10000 outpatient visits. ${ }^{10}$ Cataract formation is commonly observed as a result of direct injury to the crystalline lens by a foreign object or by blunt trauma to the globe. ${ }^{6,7}$ In most cases, the traumatic cataract interferes with the visual axis and requires cataract surgery but in some cases, a minor injury to the lens may result in a localized non-progressive lens opacity that does not require surgery. ${ }^{11}$ Damage to the zonules may also occur, causing subluxation or total displacement of the crystalline lens, ${ }^{7}$ which may also necessitate cataract surgery.

Traumatic cataract surgery is performed either as an early procedure in association with the repair of laceration in open globe injury, at early

\section{Introduction}

${ }^{1}$ Ocular Trauma and Emergency, Eye Research Center, Farabi Eye Hospital, Tehran University of Medical Sciences,

Tehran, Iran

${ }^{2}$ Tehran University of Medical Sciences, Tehran, Iran

Correspondence:

M Soleimani, Ophthalmologist, Fellowship of Anterior Segment, Eye Research Center, Farabi Eye Hospital, Tehran University of Medical Sciences, Tehran 1336616351, Iran Tel/Fax: +98 219121096496. E-mail: Soleimani_md@ yahoo.com

Received: 22 August 2016 Accepted in revised form: 24 February 2017 Published online: 14 April 2017 
days after presentation of penetrating injury, or as a late procedure in a quiet eye. If the lens capsule is ruptured and lens materials have been introduced into the anterior chamber it is preferable that lensectomy be performed as an early procedure to prevent inflammation, lens particle induced uveitis and glaucoma. Because of corneal irregularity, or associated open globe injury the power of intraocular lens (IOL) is calculated mostly by the biometry of the fellow eye.

Early procedure has the advantage of performing traumatic cataract surgery at the same admission and reducing the cost and time, while the advantages of the late procedure include performing surgery in a quiet eye, better IOL calculation after suture removal and knowing the potential for visual improvement. There is no consensus about the proper timing of traumatic cataract surgery and the influence of it on visual prognosis in the literature. So we performed the present study to compare early and late post traumatic cataract surgeries.

\section{Patients and methods}

In a randomized clinical trial, 30 eyes with traumatic cataract after open globe injury underwent early (during the first week after the trauma; Group 1) and 30 eyes underwent late cataract surgery (from the first to second month after the trauma; Group 2) (Figure 1a). All cases were observed for at least a 6-month period after the surgery. Inclusion criteria included patients older than 12-year-old with significant traumatic cataract after open globe injury. Exclusion criteria included patients with anterior capsule rupture as well as disseminated lens materials in the anterior chamber (Figure 1b), patients with hypopyon, eyes undergoing simultaneous primary repair and cataract extraction, cases that IOL implantation was not possible and patients with direct trauma to the posterior segment or with lacerations associated with vitreous prolapse.

In those cases, that IOL power calculation was not possible by the biometry of the affected eye, it was performed using the biometry of the fellow eye. The Ethics Committee of Tehran University of Medical Sciences approved the conduction of the present study and all procedures started after written informed consent was obtained following the explaining the possible consequences of the different methods of treatment to patients. All procedures were performed by the same surgeon (MBR).

All cases underwent lensectomy using vitrectomy probe through the limbal incision. We excluded those patients who may need pars plana vitrectomy from the study before randomization. They were any vitreous involvement (possibly hemorrhage, fibrin or retinal detachment) in the echography, lacerations involving more than $3 \mathrm{~mm}$ of pars plana or lacerations associated with vitreous prolapse. Each case underwent intraoperative fundoscopy after lensectomy. No case needed pars plana vitrectomy during the lensectomy procedure. Anterior capsulectomy was performed using a vitrectomy probe and anterior vitrectomy was performed in cases of posterior capsular rupture or vitreous prolapse because of zonular dehiscence. If there was a too hard nuclear part for the vitrectomy probe, we emulsified and suctioned the nucleus using a phacoemulsification handpiece. If the capsular integrity permitted, a posterior chamber IOL was implanted. In this situation, if posterior capsule was not intact, a three piece acrylic foldable a

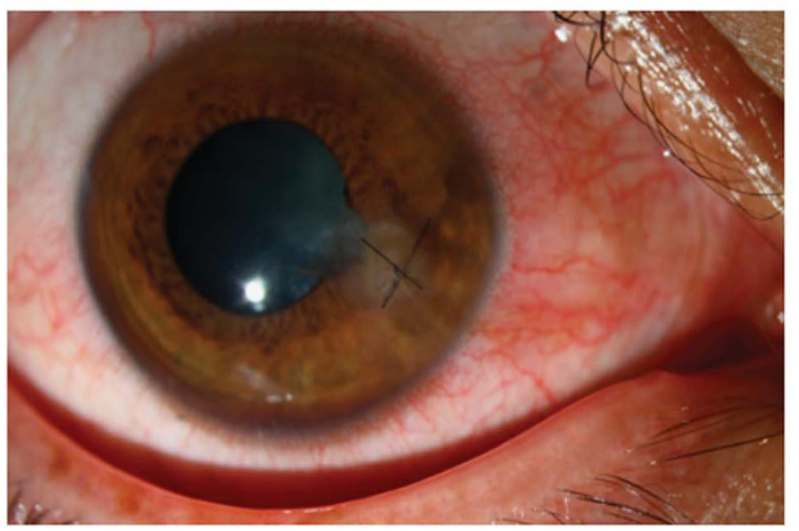

b

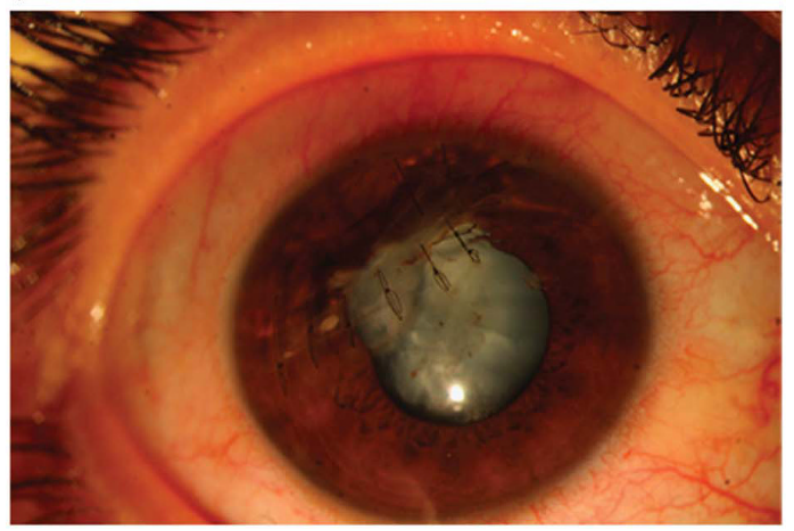

Figure 1 (a) A patient included in the study that underwent primary repair after a trauma with a metal wire when he was on work. (b) A patient who was referred with a traumatic cataract. He underwent primary repair but as can be seen in the figure lens materials were disseminated in the anterior chamber (AC), this patient was excluded, because the lens materials in AC by oneself is an indication for early cataract surgery. 
(Alcon MA60) IOL was implanted. In other situations, a one-piece foldable acrylic (Alcon SA60) IOL was implanted.

Patients were randomly assigned for early or late cataract extraction and IOL implantation. Randomization was based on a computer-generated sequence based on permuted block randomization method. The length of blocks varied randomly between two, four and six patients. A biostatistician performed the randomization and the sequence of randomization was concealed from the investigators.

All patients received prophylactic oral antibiotic therapy (ciprofloxacin $750 \mathrm{mg}$ twice daily for three days) following the open globe injury. (1). Following surgery, topical antibiotics, corticosteroids and cycloplegics were prescribed and then topical steroids were continued for four weeks and tapered gradually during this period.

We visited all patients at 1 week, 4 weeks and 12 weeks and 6 months after surgery. In each visit, patients were examined regarding visual acuity, intraocular pressure (IOP), anterior chamber inflammation, IOL position and posterior synechiae. In addition, posterior segment evaluation and fundoscopy were performed. Anterior chamber inflammation assessed by Standardization of Uveitis Nomenclature (SUN) Working Group grading scheme for anterior chamber cells and flare and grade $\geq 2+$ was considered significant. ${ }^{12}$ BCVA (best-corrected contact lens visual acuity) of both eyes was evaluated in $\log$ MAR at six months of follow up visits. Another surgeon who did not know timing of the surgery (MS) performed this evaluation.

Sample size was calculated as 30 eyes to have a power of $90 \%$ to detect a difference of $0.2 \log$ MAR in BCVA between the two groups when the standard deviation of BCVA was assumed to be $0.24 \log$ MAR and the type I error was 0.05 for two-sided test. To assess for the normal distribution of data we used Q-Q plot and KolmogorovSmirnov test. To describe data, we used mean, standard deviation, median and range. To compare the results between two groups we used $t$-test, Mann-Whitney, Fisher exact, and $\chi^{2}$-tests. All statistical methods were performed using SPSS software (IBM Corp., Armonk, NY, USA, Released 2013. IBM SPSS Statistics for Windows, Version 22.0. IBM Corp.). P-values $<0.05$ were considered statistically significant.

\section{Results}

There were 30 patients in group I with a mean age of 31.1 years (range: $12-67$ years). Thirty patients with mean age of 31.8 years (range: 12-69 years) were in group II.

Although most of patients in each group were male, there was no significant difference between two groups (Table 1). Intraoperative complications including
Table 1 Baseline characteristics of patients entering the study

\begin{tabular}{|c|c|c|c|c|}
\hline \multirow[t]{2}{*}{ Parameter } & \multirow[t]{2}{*}{ Total } & \multicolumn{2}{|c|}{ Groups } & \multirow[t]{2}{*}{ P-value } \\
\hline & & I & $I I$ & \\
\hline Number & 60 & 30 & 30 & \\
\hline \multicolumn{5}{|l|}{ Age } \\
\hline $\begin{array}{l}\text { Mean } \pm \text { SD } \\
\text { Median } \\
\text { (range) }\end{array}$ & $\begin{array}{c}31.4 \pm 14.4 \\
29 \\
(12 \text { to } 69)\end{array}$ & $\begin{array}{c}31.1 \pm 13.8 \\
29.5 \\
\text { (12 to } 67)\end{array}$ & $\begin{array}{c}31.8 \pm 15.1 \\
27 \\
(12 \text { to } 69)\end{array}$ & $0.859^{\mathrm{a}}$ \\
\hline \multicolumn{5}{|l|}{ Gender } \\
\hline Male & $55(91.7 \%)$ & $28(93.3 \%)$ & $27(90.0 \%)$ & $>0.99^{\mathrm{b}}$ \\
\hline Female & $5(8.3 \%)$ & $2(6.7 \%)$ & $3(10.0 \%)$ & \\
\hline \multicolumn{5}{|l|}{ Laterality } \\
\hline OD & 31 (51.7\%) & $12(40.0 \%)$ & $19(63.3 \%)$ & $0.071^{c}$ \\
\hline OS & $29(48.3 \%)$ & $18(60.0 \%)$ & $11(36.7 \%)$ & \\
\hline \multicolumn{5}{|l|}{ Axial Length } \\
\hline Mean \pm SD & $23.39 \pm 1.71$ & $23.66 \pm 2.17$ & $23.11 \pm 1.05$ & $0.216^{\mathrm{d}}$ \\
\hline $\begin{array}{l}\text { Median } \\
\text { (range) }\end{array}$ & $\begin{array}{c}23.04(21.31 \\
\text { to } 34.22)\end{array}$ & $\begin{array}{c}23.25(22.03 \\
\text { to } 34.22)\end{array}$ & $\begin{array}{c}22.99(21.31 \\
\text { to } 25.84)\end{array}$ & \\
\hline
\end{tabular}

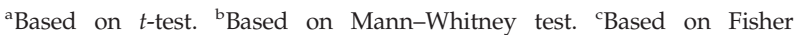
exact test. ${ }^{\mathrm{d}}$ Based on $\chi^{2}$-test.

Table 2 Best-corrected visual acuity at sixth post-operative month among patients entering the study

\begin{tabular}{lcccc}
\hline Parameter & Total & \multicolumn{2}{c}{ Groups } & P-value \\
\cline { 3 - 4 } & & $I$ & $I I$ & \\
\hline BCVA & & & & \\
$\quad \begin{array}{l}\text { Mean } \pm \text { SD } \\
\text { Median }\end{array}$ & $0.15 \pm 0.2$ & $0.12 \pm 0.15$ & $0.18 \pm 0.24$ & $0.220^{\text {a }}$ \\
(range) & $0.1(0$ to 1$)$ & $0.05(0$ to 0.5$)$ & $0.1(0$ to 1$)$ & \\
\hline
\end{tabular}

Abbreviation: BCVA: Best contact lens corrected visual acuity. ${ }^{\text {Based on }}$ Mann-Whitney test.

posterior capsular rupture, anterior vitrectomy and zonulysis at the site of IOL implantation are listed in Table 2. Iris fixation of IOL using a 10-0 prolene was used for three unstable IOL in group I and one case in group II $(P=0.309)$. Three cases of zonulysis were managed using a capsular tension ring (CTR) and a Cionni ring was implanted for the last one. Table 3 shows the BCVA and refractive status in each group. There was no significant difference between the two groups in each parameter. Post-operative complications including raised IOP, significant anterior chamber inflammation $(\geq 2+$ according to Standardization of Uveitis Nomenclature (SUN) grading scale), visual axis opacity (VAO), posterior synechiae, subluxation of IOL, IOL pigment deposition are listed in Table 4. All patients with increased IOP were medically controlled except one case in group I that finally underwent shunt procedure bringing it into 
Table 3 Intraoperative complications among patients

\begin{tabular}{|c|c|c|c|c|c|c|c|}
\hline \multirow[t]{2}{*}{ Parameter } & \multirow[t]{2}{*}{ Total } & \multicolumn{2}{|c|}{ Groups } & \multirow[t]{2}{*}{ Risk Difference } & \multicolumn{2}{|c|}{$95 \%$ CI } & \multirow[t]{2}{*}{ P-value } \\
\hline & & $I$ & II & & Lower & Upper & \\
\hline \multicolumn{8}{|l|}{ IOL Position } \\
\hline Bag & $44(73.3 \%)$ & $20(66.7 \%)$ & $24(80.0 \%)$ & & & & \\
\hline Sulcus & $16(26.7 \%)$ & $10(33.3 \%)$ & $6(20.0 \%)$ & $-13.30 \%$ & $-36.30 \%$ & $9.70 \%$ & 0.250 \\
\hline Posterior capsular rupture & $14(23.3 \%)$ & $10(33.3 \%)$ & $4(13.3 \%)$ & $-20.00 \%$ & $-41.60 \%$ & $1.60 \%$ & 0.069 \\
\hline Anterior vitrectomy & $17(28.3 \%)$ & $10(33.3 \%)$ & $7(23.3 \%)$ & $-10.00 \%$ & $-33.50 \%$ & $13.50 \%$ & 0.399 \\
\hline Zonulysis & $4(6.7 \%)$ & $0(0.0 \%)$ & $4(13.3 \%)$ & $13.30 \%$ & $0.70 \%$ & $26.00 \%$ & 0.039 \\
\hline
\end{tabular}

${ }^{a}$ Based on $\chi^{2}$ or Fisher exact test, whenever appropriate.

Table 4 Post-operative complications in early and late cataract surgery groups during six month follow up

\begin{tabular}{|c|c|c|c|c|c|c|c|}
\hline \multirow[t]{2}{*}{ Parameter } & \multirow[t]{2}{*}{ Total } & \multicolumn{2}{|c|}{ Groups } & \multirow[t]{2}{*}{ Risk Difference } & \multicolumn{2}{|c|}{$95 \% C I$} & \multirow[t]{2}{*}{ P-value } \\
\hline & & $I$ & II & & Lower & Upper & \\
\hline Raised IOP after Surgery & $4(6.7 \%)$ & $3(10.0 \%)$ & $1(3.3 \%)$ & $-6.70 \%$ & $-19.70 \%$ & $6.30 \%$ & 0.309 \\
\hline Inflammation & $4(6.7 \%)$ & $1(3.3 \%)$ & $3(10.0 \%)$ & $6.70 \%$ & $-6.30 \%$ & $19.70 \%$ & 0.309 \\
\hline Visual axis opacity (VAO) & $9(15.0 \%)$ & $3(10.0 \%)$ & $6(20.0 \%)$ & $10.00 \%$ & $-8.60 \%$ & $28.60 \%$ & 0.286 \\
\hline Posterior Synechiae & $7(11.7 \%)$ & $2(6.7 \%)$ & $5(16.7 \%)$ & $10.00 \%$ & $-6.70 \%$ & $26.70 \%$ & 0.235 \\
\hline Subluxation of IOL & $2(3.3 \%)$ & $0(0.0 \%)$ & $2(6.7 \%)$ & $6.70 \%$ & $-2.60 \%$ & $15.90 \%$ & 0.155 \\
\hline IOL pigment deposition & $5(8.5 \%)$ & $3(10.0 \%)$ & $2(6.9 \%)$ & $-3.10 \%$ & $-17.90 \%$ & $11.60 \%$ & 0.675 \\
\hline
\end{tabular}

${ }^{a}$ Based on $\chi^{2}$ or Fisher exact test, whenever appropriate.

control. There was not any case of sympathetic ophthalmia in both groups.

\section{Discussion}

This study was a prospective randomized control study that performed with enrollment of 60 patients with traumatic cataract after open globe injury. The purpose of the study was to determine the proper time for traumatic cataract surgery. A vast majority of traumatic ocular injuries are associated with traumatic cataract. ${ }^{5,6}$ Ocular trauma mainly involves children and young men. ${ }^{79}$ So it is reasonable to perform traumatic cataract surgery as an early procedure in this group to make the period of visual rehabilitation shorter and starting the amblyopic therapy sooner with the help of modern surgical techniques that have yielded good anatomic and functional outcomes in the treatment of traumatic eye injuries recently. ${ }^{13,14} \mathrm{On}$ the other hand, late cataract surgery could permit to better IOL calculation, probably operating in an eye with less corneal edema and inflammation and better evaluation of the traumatic cataract. One study has been performed by Shah et al. ${ }^{15}$ to determine the effect of interval between the time of injury and time of intervention on the final visual outcome in cases of traumatic cataract. Their study was a prospective cohort and performed between January 2003 and December 2009. In patients with corneal laceration, traumatic cataract surgery performed as a secondary procedure.
This group of patients had a significant higher postoperative inflammation and lower final BCVA. They concluded that it is better to perform the traumatic cataract surgery as an early procedure because of lower complication rate as well as sooner and better visual rehabilitation. ${ }^{15}$ Some other authors like Memon et al, ${ }^{16}$ advocate secondary procedure in the absence of fragmentized or intumescent traumatic cataract to manage traumatic cataract. Reasons that Memon et al, ${ }^{16}$ mention are better assessment of visual improvement after surgery, more accurate IOL calculation and less postop inflammation. Also Agarwal et a $l^{17}$ have suggested that in the presence of severe corneal damage and edema it is better to postpone lensectomy for better visualization during surgery. There are some special situations which ocular trauma is associated with the rupture of anterior capsule and presence of lens material in anterior chamber. The possibility of IOP rise and prolonged inflammation, which may cause anterior and posterior synechiae, is higher in this situation. The longer existence of synechiae the more difficult the surgery will be. Therefore, the repair of the traumatic wound and lensectomy is better to be done at the same session. ${ }^{12}$ On the other hand, it is possible that the patient with ocular trauma would have small, peripheral opacity in crystalline lens that does not obscure visual axis. It is better to follow up these cases with regular visits to determine that lensectomy is a good choice for them or not. 
In the present study, we tried to make a model to find the proper time of traumatic cataract surgery. We did not include patients who underwent cataract surgery between the first week and the first month, because the "early" time (the first week after trauma) is usually in the same admission period, after one week, up to one month, one could possibly follow the patient in the hope that the inflammation and edema would be improved, also there would be a time lag after primary admission. In this study, the mean BCVA 6 months after surgery was relatively good because associated ocular injuries excluded based on exclusion criteria. Kuhn et al ${ }^{18}$ have stated that any traumatic injury to ocular tissue in patients with traumatic cataract can lead to a decreased post-operative BCVA. Srivastava et al. ${ }^{19}$ have reported that demographic variables such as age, sex, and the time of intervention have no association with post-operative BCVA, similar to our results. We found that at 6 months post operative the BCVA showed no difference among patients in two groups. In addition, intra- and postoperative complications were not different between early and late procedure groups.

Posterior capsular rupture was not statistically different between group I and group II $(P=0.069)$. Although not quite reaching statistical significance due to small numbers, there is a trend towards a greater risk (a 20\% increase in Table 3) of posterior capsule rupture in group 1 .

We found more zonulysis in group II, possibly because during the early procedures, zonulysis had been considered possibly as posterior capsular rupture and had been managed with anterior vitrectomy (probably because of a lower vision during early surgery). Finally, the rate of anterior vitrectomy was not different between early and late procedure groups $(P=0.399)$. Although there are some difficulties during operation such as intraoperative corneal edema that may compromise vision during the operation, the rate of complications was not different between the two groups. The study had some limitations; The follow-up period was 6 months in this study, we need more studies with longer follow-ups in the future. The mean 6 months post-operative BCVA was relatively good among our patients because of strict exclusion criteria such as patients with associated ocular trauma and released lens materials in anterior chamber were excluded from the study, so one of our limitations is that we may not be able to generalize our results to all traumatic cataract patients. The low number of cases in the present study is one of the limitations. In addition, we included the patients undergoing cataract surgery in the first week but not in the primary repair session. Although this was to preserve ethical purposes, one could think about the results of primary repair and lensectomy in the same session. In addition, we only included patients undergoing IOL implantation, thus we do not know the detailed results in aphakic eyes. Finally, according to this study (1) There was no difference regarding visual outcomes between the two groups (2) There was no difference regarding intra- or post- operative complications between the groups (3) So, early and late intervention could be considered equally according to the situation of each case and the treatment environment, (4) However, we need more trials addressing mentioned limitations to say the correct timing of traumatic cataract surgery.

\section{Summary}

What was known before

- In traumatic cataract after open globe injuries, when anterior capsule is open as well as there are released lens materials in the anterior chamber, it is necessary to do early cataract surgery; timing of traumatic cataract surgery after open globe injuries is a controversial item.

What this study adds

- In traumatic cataract surgery and IOL implantation after open globe injuries, there is no difference between early and late procedure, regarding the post-surgical BCVA and prominent intraoperative and post-operative complications.

\section{Conflict of interest}

The authors declare no conflict of interest.

\section{References}

1 Tabatabaei SA, Soleimani M, Behrooz MJ, Sheibani K. Systemic oral antibiotics as a prophylactic measure to prevent endophthalmitis in patients with open globe injuries in comparison with intravenous antibiotics. Retina 2016; 36(2): 360-365.

2 Cabalag MS, Wasiak J, Syed Q, Paul E, Hall AJ, Cleland H. Early and late complications of ocular burn injuries. J Plast Reconstr Aesthet Surg 2015; 68(3): 356-361.

3 Katz J, Tielsch JM. Lifetime prevalence of ocular injuries from the Baltimore Eye Survey. Arch Ophthalmol 1993; 111(11): 1564-1568.

4 Klopfer J, Tielsch JM, Vitale S, See LC, Canner JK. Ocular trauma in the United States. Eye injuries resulting in hospitalization, 1984 through 1987. Arch Ophthalmol 1992; 110(6): 838-842.

5 Jones WL. Traumatic injury to the lens. Optom Clin 1991; 1(2): 125-142.

6 Macewen CJ. Eye injuries: a prospective survey of 5671 cases. Br J Ophthalmol 1989; 73(11): 888-894.

7 Dannenberg AL, Parver LM, Brechner RJ, Khoo L. Penetration eye injuries in the workplace. The National Eye Trauma System Registry. Arch Ophthalmol 1992; 110(6): 843-848.

8 Slusher MM, Greven CM, Yu DD. Posterior chamber intraocular lens implantation combined with lensectomy- 
vitrectomy and intraretinal foreign-body removal. Arch Ophthalmol 1992; 110(1): 127-129.

9 Pieramici DJ, Sternberg Jr P, Aaberg Sr TM, Bridges Jr WZ, Capone Jr A, Cardillo JA et al. A system for classifying mechanical injuries of the eye (globe). The Ocular Trauma Classification Group. Am J Ophthalmol 1997; 123(6): 820-831.

10 Rumelt S, Rehany U. The influence of surgery and intraocular lens implantation timing on visual outcome in traumatic cataract. Graefes Arch Clin Exp Ophthalmol 2010; 248(9): 1293-1297.

11 Moisseiev J, Segev F, Harizman N, Arazi T, Rotenstreich Y, Assia EI. Primary cataract extraction and intraocular lens implantation in penetrating ocular trauma. Ophthalmology 2001; 108(6): 1099-1103.

12 Agrawal R, Keane PA, Singh J, Saihan Z, Kontos A, Pavesio $\mathrm{CE}$. Classification of semi-automated flare readings using the Kowa FM 700 laser cell flare meter in patients with uveitis. Acta Ophthalmol 2016; 94(2): e135-e141.

13 Ryan SJ, Allen AW. Pars plana vitrectomy in ocular trauma Am J Ophthalmol 1979; 88(3 Pt 1): 483-491.

14 Rubsamen PE, Cousins SW, Winward KE, Byrne SF. Diagnostic ultrasound and pars plana vitrectomy in penetrating ocular trauma. Ophthalmology 1994; 101(5): 809-814.

15 Shah MA, Shah SM, Shah SB, Patel UA. Effect of interval between time of injury and timing of intervention on final visual outcome in cases of traumatic cataract. Eur J Ophthalmol 2011; 21(6): 760-765.

16 Memon MN, Narsani AK, Nizamani NB. Visual outcome of unilateral traumatic cataract. J Coll Physicians Surg Pak 2012; 22(8): 497-500.

17 Agarwal A, Ashok Kumar D. How to manage the complications of a traumatic cataract, ocular surgery news U.S. Edition. Available at: http://www.healio.com/ophthalmology/ cataract-surgery/news/print/ocular-surgery-news/\% 7Ba5742be5-6e8a-43c8-a241-1598aac8449a\%7D/how-tomanage-the-complications-of-a-traumatic-cataract (last accessed on 04 August 2016).

18 Kuhn F, Morris R, Witherspoon CD, Mester V. The Birmingham Eye Trauma Terminology system (BETT). J Fr Ophtalmol 2004; 27(2): 206-210.

19 Srivastava U, Lalramhluni R, Preeti Rawat V. Clinical evaluationof post traumatic cataract in tertiary care hospital. Int J Sci Res Pub 2014; 4(10): 1-6. 